\title{
Awareness and Opinions of Inhabitants on Vivax Malaria in Two Endemic Areas, Gyeonggi-do, Korea
}

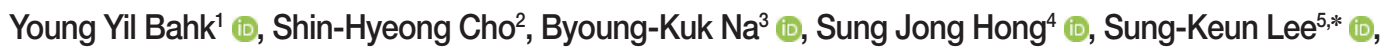 \\ Tong-Soo Kim ${ }^{4,6, *}$ iD \\ 'Department of Biotechnology, College of Biomedical and Health Science, Konkuk University, Chungju 27478, Korea; '2Noul Co. LTD., Yongin 16942, \\ Korea; ${ }^{3}$ Department of Parasitology and Tropical Medicine, and Institute of Health Sciences, Gyeongsang National University College of Medicine, \\ Jinju 52727, Korea, and Department of Convergence Medical Science, Gyeongsang National University, Jinju 52727, Korea; ${ }^{4}$ Convergence \\ Research Center for Insect Vectors, Incheon National University, Incheon 22012, Korea; ${ }^{5}$ Department of Pharmacology, Inha University School of \\ Medicine, Incheon 22212, Korea; ${ }^{6}$ Department of Tropical Medicine, Inha University School of Medicine, Incheon 22212, Korea
}

\begin{abstract}
The incidence of vivax malaria in Korea was reduced to a low plateau. For successful elimination of vivax malaria, socio-behavioral changes in the communities are essential. This study aimed to figure out awareness of the inhabitants on the vivax malaria endemicity. The 407 participants including vivax malaria patients and uninfected inhabitants in Gimpo- and Paju-si, Gyeonggi-do, known as high-risk areas in Korea. We used a community-based study design and non-probability sampling method using primary data. Except for the perception about the public health facilities' capability to cope with anti-malaria programs, the 2 groups of participants shared the same level of awareness about public promotional and educational measures and opinions for malaria elimination from the community. Thus, our future goals for malaria prevention and elimination are to develop more active and well-organized community-based education and evaluation programs collaborating with the community healthcare authorities and local governments.
\end{abstract}

Key words: Vivax malaria, endemicity, awareness, education, public health

The World Health Organization (WHO) defines malaria elimination as reduction in malaria cases to 0 in a defined geographical area as a result of deliberate efforts [1]. In Korea, malaria incidence per 100,000 people, ranks first position among Organization for Economic Co-operation and Development Countries, followed by Mexico with 0.6. It was expected that Korea eliminate vivax malaria by 2023 [2]. To achieve this goal, the Korea Disease Control and Prevention Agency (KDCA) has launched a 'Five-Year Action Plan for Malaria Elimination (2019-2023)'. However, it proceeded slowly and was partially successful. Despite being preventable and treatable, vivax malaria significantly influences the public health of residents in adjacent regions to the demilitarized zone between South and North Korea [3]. Even though malaria decreased dramatically in Korea, its elimination was not so easily come to fruit [4]. Malaria elimination strategies must involve

- Received 28 September 2021, revised 11 October 2021, accepted 12 October 2021.

*Corresponding authors (sungkeun@inha.ac.kr; tongsookim@inha.ac.kr)

(c) 2021, Korean Society for Parasitology and Tropical Medicine

This is an Open Access article distributed under the terms of the Creative Commons Attribution Non-Commercial License (https://creativecommons.org/licenses/by-nc/4.0) which permits unrestricted non-commercial use, distribution, and reproduction in any

medium, provided the original work is properly cited. profound community commitment at behavioral level, beginning with the most affected marginalized populations. Advancing from malaria control to elimination would require more prompt and efficacious community commitment to ensure that the communities are equally enthusiastic and participate adequately in the elimination programs. Local and focused approaches are required to engage and target the highrisk areas of malaria. In fact, malaria elimination strategies must consider the volatility of malaria including lack of precautionary measures taken by villagers as they repeatedly enter malaria-prone areas.

The link between health and socio-demographic status has well been studied. In previous studies, health education was the most important determinant of project outcomes [5]. Consequently, reducing public stigma toward infectious diseases, such as malaria, and improving public health knowledge represent a major challenge to enable early interventions. This study aimed to evaluate the participants' awareness of the need to prevent and eliminate malaria from their residing community, especially malaria programs and the role of education.

This study was conducted in 2 cities (Gimpo and Paju) in 
Gyeonggi-do from May to October 2020. The study procedures concerning human sample collection, laboratory investigation, interviews, and questionnaire were reviewed and approved by the Institutional Review Board of Inha University (Approval No. 2020-04-004). This study was conducted according to the principles expressed in the 1964 Helsinki Declaration. The written informed consent was obtained all from the study participants who were informed of their right to refuse to participate and/or to withdraw from the study at any time. For data collection, an interviewer-administrated questionnaire was prepared with reference to the previous literatures. In this study, 406 participants (380 uninfected inhabitants and 26 vivax malaria patients) were interviewed, and the data collected were analyzed. After the questionnaire, the unanswered and dependent data were excluded from the analysis and the statistical package, SPSS program 21.0 version (Statistical Package for Social Science, IBM, Armonk, New York, USA) and R-studio, were used for data coding and cleaning processes. The statistical procedures in this study were performed by ST-Research, Inc. (Busan, Korea).

The socio-demographic characteristics of the participants in this study is presented in Table 1 . The dependent variables in- cluded promotional measures to prevent malaria infection by the regional community were assessed using 4 malaria publicity-related questions (Table 2). The questions used to assess these issues were 1) have you ever heard of malaria publicity? 2) are you aware of means to gain experience to cognize malaria publicity? 3) what are effective methods for malaria publicity? and 4) are you willing to participate in future malaria publicity programs? Multiple choices were provided for some questions in the questionnaire.

When respondents asked whether you have ever heard of malaria publicity, 160 (43.7\%) responded positively (uninfected inhabitant (UI) group 149/340 (43.8\%), patient (P) group) $11 / 26(42.7 \%))$. Almost the same proportion of respondents $(165,45.1 \%)$ responded that they had never heard $(152,41.5 \%)$ or not heard $(13,3.6 \%)$ of malaria publicity. Therefore, similar number of respondents responded negatively in both groups. Responses regarding means to gain experience to cognize malaria publicity, the most common source was leaflets provided by public health facilities (155/393 (39.4\%), UI group: 149/367 (40.6\%), P group: 6/26 (23.1\%). However, $46.8 \%$ (184/393) of the respondents in this study reported that they had never witnessed any publicity for ma-

Table 1. Socio-demographic characteristics of respondents in Paju- and Gimpo-si, Gyeonggi-do, Korea, 2020 ( $n=407)$

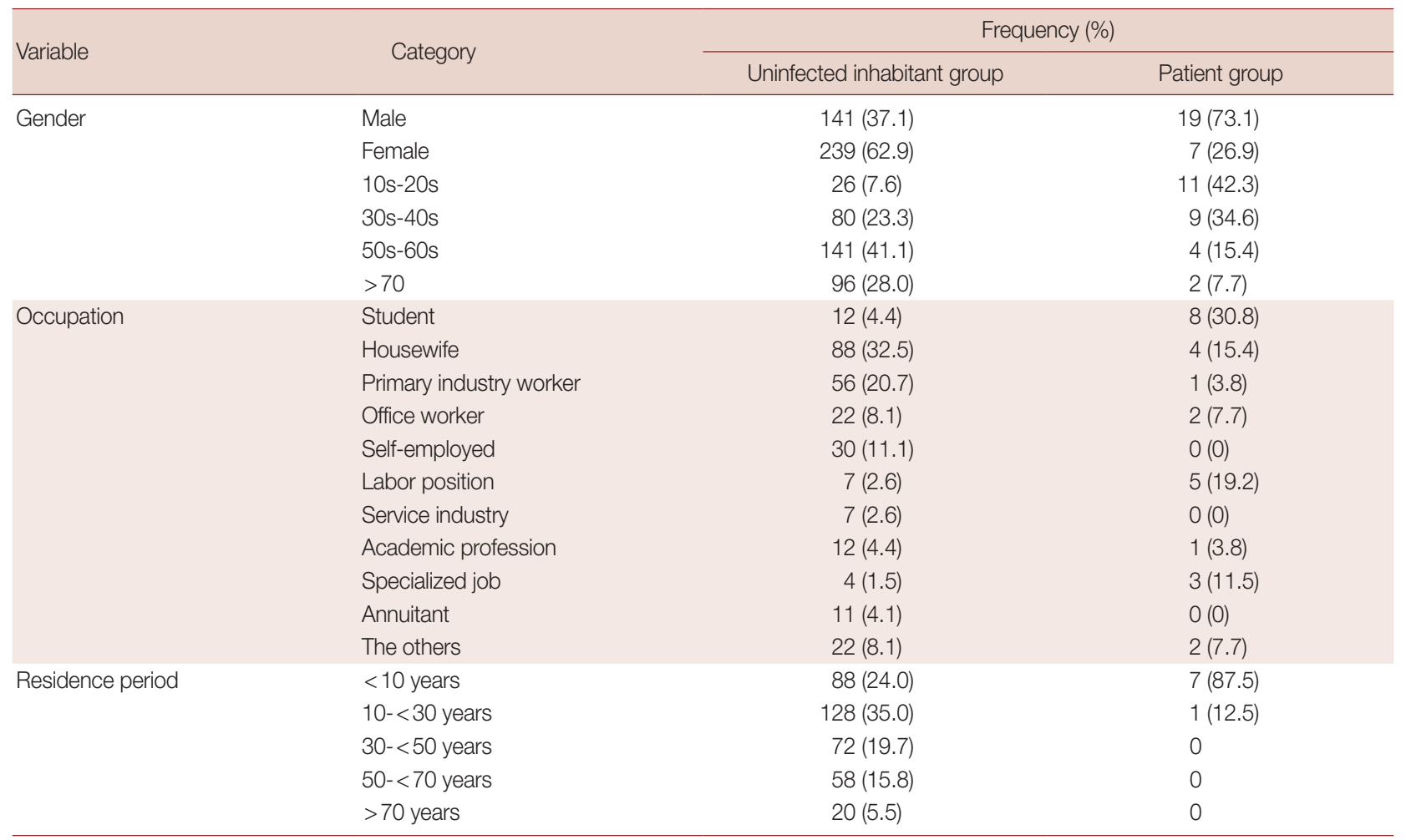


laria prevention and elimination (UI group: 169 (46.0\%), P group: 15 (57.7\%)). Responses regarding the effective methods for malaria publicity, approximately one-third of the study respondents (139/394 (35.3\%), UI group: 134/370 (36.2\%), P group: 5/26 (20.8\%)) mentioned leaflets provided by public health facilities, followed by regular education at town halls, senior citizen centers, etc., by public health facilities (111 (28.2\%), UI group: 109 (29.5\%), P group: 2 (8.3\%)), public broadcasting by regional community (102 (25.9\%), UI group: 93 (25.1\%), P group: $9(37.5 \%))$, and street banner (92 (23.4\%), UI group: 83 (22.4\%), P group: 9 (37.5\%)). According to this result, not only the simple street campaign that has been performed in the regional community so far, but also various and carefully orchestrated publicity campaign are required. In Zhangjiagang city, knowledge of the prevention and control of malaria was propagandized through WeChat SNS among these personnel, and the public awareness of malaria control, investigated by questionnaires before and after the publicity through WeChat, had improved significantly [6]. More than $70 \%$ of the respondents in this study expressed their willingness to participate in public education programs to prevent and eliminate malaria in the future. However, very few respondents were definitely determined to participate (67/367 (18.3\%) in UI group only).

When the study respondents were asked how to eliminate malaria in their residing community (Table 3), the most common necessary measure mentioned was use of mosquito repellents (75.1\%), followed publicity service of prevention and treatment against malaria infection (41.5\%), and rapid diagnosis and treatment (24.4\%). The same participants in this study reported previously that the 3 common preventive measures were not getting bitten by mosquitoes, wearing longsleeved and bright-colored clothing, and being cautious when visiting malaria-prone regions. The responses about malaria prevention measures differed significantly between the UI and P groups. In this study, half of the P group respondents reported that the most necessary measure to prevent and eliminate malaria is rapid diagnosis and treatment. When asked about the most influential agencies providing anti-malaria service, both groups (UI group 63.1\%, P group 88.5\%) cited local gov-

Table 2. Response to promotional measures to prevent malaria infection, promoted by regional community

\begin{tabular}{|c|c|c|}
\hline \multirow{2}{*}{ Question and options } & \multicolumn{2}{|c|}{ Frequency (\%) } \\
\hline & Uninfected inhabitant group & Patient group \\
\hline Have you ever heard of malaria publicity? & $n=340$ & $n=26$ \\
\hline Never heard & $143(39.4)$ & $9(34.6)$ \\
\hline Not heard & $12(3.5)$ & $1(3.8)$ \\
\hline I don't know. & $45(13.2)$ & $5(19.2)$ \\
\hline Heard & $131(38.5)$ & $11(42.3)$ \\
\hline Frequently heard & $18(5.3)$ & $0(0)$ \\
\hline Experience means to cognize malaria publicity* & $\mathrm{n}=367$ & $\mathrm{n}=26$ \\
\hline Public broadcasting by regional community (Apartment managing office, etc.) & $24(6.5)$ & $3(11.5)$ \\
\hline Leaflet provided by public health facilities & $149(40.6)$ & $6(23.1)$ \\
\hline Street campaign & $27(7.4)$ & $1(3.8)$ \\
\hline Banner & $33(9.0)$ & $2(7.7)$ \\
\hline Never seen & $169(46.0)$ & $15(57.7)$ \\
\hline Effective method for malaria publicity ${ }^{\star}$ & $\mathrm{n}=370$ & $\mathrm{n}=24$ \\
\hline Public broadcasting by regional community (Apartment etc.) & $93(25.1)$ & $9(37.5)$ \\
\hline Leaflet provided by public health facilities & $134(36.2)$ & $5(20.8)$ \\
\hline Street campaign & $48(13.0)$ & $4(16.7)$ \\
\hline Banner & $83(22.4)$ & $9(37.5)$ \\
\hline Regular education at town hall, senior citizen center, etc., by public health facilities & $109(29.5)$ & $2(8.3)$ \\
\hline I don’t know. & $48(9.3)$ & $1(4.2)$ \\
\hline Willingness to participate in future malaria education* & $\mathrm{n}=367$ & $n=26$ \\
\hline Not participate & $14(3.8)$ & $3(11.5)$ \\
\hline I don’t know. & $88(24.0)$ & $4(15.4)$ \\
\hline Participate if possible & $198(54.0)$ & $19(73.1)$ \\
\hline Definitely participate & $67(18.3)$ & $0(0)$ \\
\hline
\end{tabular}

${ }^{*}$ Respondents can choose multiple options. 
Table 3. Study respondents' opinions on how to eliminate malaria in community

\begin{tabular}{|c|c|c|}
\hline \multirow{2}{*}{ Question and options } & \multicolumn{2}{|c|}{ Frequency (\%) } \\
\hline & Uninfected inhabitant group & Patient group \\
\hline The most necessary measure to prevent and eliminate malaria & $\mathrm{n}=371$ & $n=26$ \\
\hline Promotion service of prevention and treatment against malaria infection & $154(41.5)$ & 9 (34.6) \\
\hline Use mosquito repellents & $208(56.1)$ & $10(38.5)$ \\
\hline Rapid diagnosis and treatment & $84(22.6)$ & $13(50.0)$ \\
\hline Personal protection & $61(16.4)$ & $3(11.5)$ \\
\hline Outbreak report of malaria & $29(7.8)$ & $0(0)$ \\
\hline The main agent of anti-malaria service* & $n=374$ & $n=26$ \\
\hline National institution & $122(32.6)$ & $7(26.9)$ \\
\hline Research institute such as Institute of health and environment & $67(17.9)$ & $3(11.5)$ \\
\hline Local government and public local health center & $236(63.1)$ & $23(88.5)$ \\
\hline Clinic and hospital & $31(8.3)$ & $0(0)$ \\
\hline Educational institutes such as Universities & $9(2.4)$ & $0(0)$ \\
\hline By oneself & $38(10.2)$ & $0(0)$ \\
\hline National measures for prevention and elimination of malaria* & $\mathrm{n}=374$ & $n=26$ \\
\hline Sustained promotion for malaria prevention through mass communication & $126(33.7)$ & $11(42.3)$ \\
\hline Expended education on malaria prevention & $104(27.8)$ & $6(23.1)$ \\
\hline Expanded management service such mosquito control and quarantine, etc. & $185(49.5)$ & $8(30.8)$ \\
\hline Strengthen promotion service for personal protection activities & $15(4.0)$ & $0(0)$ \\
\hline Rapid diagnosis and treatment & $48(12.8)$ & $9(34.6)$ \\
\hline Public health facilities' capability to cope with anti-malaria service & $n=370$ & $n=26$ \\
\hline Being very unresponsible & $36(9.7)$ & $0(0)$ \\
\hline Being slightly unresponsible. & $49(13.2)$ & $2(7.7)$ \\
\hline I don't know. & $148(40.0)$ & $10(38.5)$ \\
\hline Being responsible & $112(30.3)$ & $12(46.2)$ \\
\hline Being well responsible & $25(6.8)$ & $5(7.1)$ \\
\hline
\end{tabular}

${ }^{*}$ Respondents can choose multiple options.

ernments and local public health facilities, followed by the national institution (UI group 32.6\%, P group 26.9\%) and research institute, such as Institute of health and environment (UI group17.9\%, P group11.5\%). Engagement and participation of local health authorities, such as the local Public Health Center (PHC) and the local government, play a critical role in successfully controlling and eliminating communicable diseases [7]. In fact, rural residents in Korea had little access to health care services because most medical facilities were built in large cities. Thus, the Korea Government had prioritized establishing a national and local health promotion program long time ago [8]. Nowadays, Korea has a high coverage of health facilities. However, malaria has not been eliminated from Korea despite of the large reduction of malaria burden (>87\%) [9]. This indicates the modernized health care systems with strong surveillance systems that are less likely to report malaria cases. In Korea, the clinicians should report malaria cases by law to the local PHC. PHCs must periodically report cases to the KCDA through the infectious disease surveil- lance system [10].

In this study, approximately half of the respondents in the UI group (49.5\%) reported that expanded management services such as mosquito control and quarantine were the primary national measures taken to prevent and eliminate malaria. Next, sustained promotion for malaria prevention through mass communication (33.7\%) and expanded education programs on malaria prevention $(27.8 \%)$ were conducted. Less attention was paid to personal protection activities and rapid diagnosis and treatment. However, in the P group, the primary national measures were sustained through mass communication (42.3\%) and rapid diagnosis and treatment (34.6\%), followed by expanded management service (30.8\%) and the expanded education programs (23.1\%). Public educational campaigns may have beneficial effects on vector control within the communities by teaching the public how to maintain or eliminate these types of habitats to prevent mosquitoes from completing their development cycle [11]. Educational programs do not always have an immediate effect. Large-scale 
source reduction efforts might not noticeably and immediately decrease the mosquito population compared to insecticide spray. However, community participation is essential for developing long-term, low-cost, sustainable programs [12]. Thus, our future goals for malaria prevention and elimination are to develop and evaluate more active and well-organized community-based education and publicity programs using community healthcare authorities and local government.

Finally, regarding the public health facilities' capability to cope with anti-malaria service, $51.8 \%$ of the respondents responded negatively (UI group 52.2\%, P group 46.2\%). Among respondents in the UI group, scores for the question about the public health facilities' capability ranged from 0 to 5 with an average of $3.11 \pm 1.042$. However, in the P group, the minimum score was $3.54 \pm 0.76$. The difference in scores between the $\mathrm{UI}$ and $\mathrm{P}$ groups was significant $(P<0.05)$. The readiness of public health facilities in Korea for malaria control was associated with a dramatic decline ( $>87 \%)$ in the malaria incidence to a low plateau $[4,9]$. Service readiness refers to the overall capacity of health facilities to provide health service measured with the availability of basic equipment, standard precautions for infection prevention, diagnostic capacity and essential medicines [13]. Currently, the health services for malaria elimination in Korea have a sufficiently experienced status but of the best choice for achieving the final goal, highlighting the need for new strategies to overcome the new challenges. Thus, to eliminate malaria, each community member should be provided with affordable information from of national and local authorities. The efficient flow of information through several communication channels could be more capable of eliminating the current malaria endemic in Korea. There is also the additional benefit of empowering the community members to recognize mosquito habitats, transmission of disease, and personal protection [14]. The information can be essential to help reduce potential habitats and sustain source reduction efforts within the community. This can be accomplished by better understanding the sociology and determinants within the community, as these can greatly affect behaviors within a specific community [15].

Designing a sustainable and effective strategy for disease control and elimination is a challenge. Both social promotion and public education were beneficial in improving knowledge and attitudes toward infectious diseases. This study was carried out to explore the participants' awareness of the need to prevent and eliminate malaria from their residing community, es- pecially malaria publicity and education and role of national and local health authorities. Health has been a continuous focus of policy makers. Therefore, it is important to precisely understand the channels and mechanisms that improve health, for efficient resource allocation. Education and publicity clearly affect health through 2 of its most important components: disease detection and health management. This reminds policymakers of the importance of keeping sufficient and sustained attention on malaria elimination.

\section{ACKNOWLEDGMENT}

This study was supported by funding from the Korea Disease Control and Prevention Agency (KDCA), Korea and by funding from the National Research Fund (NRF-2017M3A9B8069530, NRF-2020R1F1A1070882), Korea.

\section{CONFLICT OF INTEREST}

The authors declare that they have no conflicts of interest regarding the publication of this article.

\section{REFERENCES}

1. World Health Organization. From Malaria Control to Malaria Elimination: a Manual for Elimination Scenario Planning. Geneva, Switzerland. 2014. http://mesamalaria.org/resource-hub/ malaria-control-malaria-elimination-manual-elimination-scenario-planning

2. World Health Organization. Republic of Korea-WHO Country Cooperation Strategy 2019-2023. Geneva, Switzerland. World Health Organization Western Pacific Region, Korea Ministry of Health and Welfare. 2019. https://apps.who.int/iris/handle/ $10665 / 279858$

3. Kim JH, Lim AY, Cheong HK. Malaria incidence of the regions adjacent to the demilitarized zone in the Democratic People's Republic of Korea, 2004-2016. J Korean Med Sci 2019; 34: e227. https://doi.org/10.3346/jkms.2019.34.e227

4. Bahk YY, Lee HW, Na BK, Kim J, Jin K, Hong YS, Kim TS. Epidemiological characteristics of re-emerging vivax malaria in the Republic of Korea (1993-2017). Korean J Parasitol 2018; 56: 531543. http://doi.org/10.3347/kjp.2018.56.6.531

5. Kim Y, Radoias V. Education, individual time preference, and asymptomatic disease detection. Soc Sci Med 2016; 150: 15-22. https://doi.org/10.1016/j.socscimed.2015.11.051

6. Xue-Dong W, Ling J, Feng H, Bin L, Yi R. Role of WeChat publicity in control of imported malaria from abroad in timber industry. Zhonggus Xue Xi Chong Bing Zhi Za Zhi 2018; 30: 688-690 
(in Chinese). https://doi.org/10.16250/j.32.1374.2018202

7. Atkinson JA, Vallely A, Fitzgerald L, Whittaker M, Tanner M. The architecture and effect of participation: a systematic review of community participation for communicable disease control and elimination. Implication for malaria elimination. Malar J 2011; 10: 225. https://doi.org/10.1186/1475-2875-10-225

8. Kwak C, Ko Y. Historical overview of community health practitioners in Korea. Public Health Nurs 2015; 32: 161-169. https:// doi.org/10.1111/phn.12143

9. Sahu M, Tediosi F, Noor AM, Aponte JJ, Fink G. Health systems and global progress towards malaria elimination, 2000-2016. Malar J 2020; 19: 141. https://doi.org/10.1186/s12936-020-03208-6

10. Korean Agency for Diseases Control and Prevention. Infectious Diseases Surveillance System. [Internet]. Available from: https:// is.cdc.go.kr/

11. Swaddiwudhipong W, Chaovakiratipong C, Nguntra P, Koonchote S, Khumklam P, Lerdlukanavonge P. Effect of health education on community participation in control of dengue hemorrhagic fever in an urban area of Thailand. Southeast Asian J Trop
Med Public Health; 23: 200-206. https://pubmed.ncbi.nlm.nih. gov/1439971/

12. Gubler DJ, Clark GG. Community involvement in the control of Aedes aegypti. Acta Trop 1996; 61: 169-179. https://doi.org/10. 1016/0001-706x(95)00103-1

13. World Health Organization. Service Availability and Readiness Assessment (SARA): an Annual Monitoring System for Service Delivery: Reference Manual. Geneva, Switzerland. World Health Organization. https://apps.who.int/iris/handle/10665/149025

14. Lloyd LS, Winch P, Ortega-Canto J, Kendall C. Results of a community-based control program in Merida, Yucatan, Mexico. Am J Trop Med Hyg 1992; 46: 635-642. https://doi.org/10.4269/ajtmh.1992.46.635

15. Healy K, Hamilton G, Crepeau T, Healy S, Unlu I, Farajollahi A, Fonseca DM. Integrating the public in mosquito management: active education by community peers can lead to significant reduction in peridomestic container mosquito habitats. PLoS One 2014; 9: e108504. https://doi.org/10.1371/journal.pone.0108504 